\title{
THE WESTERN CHRISTMAS MEETING OF THE SOCIETY AND THE MEETING OF THE SOUTHWESTERN SECTION
}

The twenty-fourth Western meeting of the Society with which was combined the eighteenth regular meeting of the Southwestern Section was held in Kansas City, on Tuesday and Wednesday, December 29 and 30, 1925. The total attendance at the meeting was about 140 , and included the following 103 members:

E. F. Allen, E. S. Allen, F. E. Allen, Archibald, G. N. Armstrong, Ashton, Atchison, R. W. Babcock, Wealthy Babcock, F. L. Black, Brenke, Cairns, Cajori, Calloway, A. D. Campbell, Carmichael, C. C. Carter, Chittenden, Coolidge, Court, Crathorne, Daniells, Dederick, De Long, Denton, E. L. Dodd, Dresden, Dunkel, Edington, Epperson, Everett, Feltges, Fields, Finkel, Fleet, Focke, Gaba, Garrett, Gerst, Gorrell, Gossard, Gouwens, Harshbarger, Hassler, Hazlett, E. R. Hedrick, Hildebrandt, Horton, J. C. Hughes, Huntington, Hyde, Byron Ingold, Louis Ingold, Dunham Jackson, Jamison, E. H. Jones, H. E. Jordan, Kline, Laves, F. P. Lewis, Lubben, Luby, Lunn, William Marshall, H. H. Marvin, J. V. McKelvey, Mirick, U. G. Mitchell, R. L. Moore, Mossman, F. R. Moulton, Muehlman, Murnaghan, Osborn, Pennell, Pettit, E. C. Phillips, Pierce, Pierpont, P. E. Remington, Rider, H. L. Rietz, Risley, Roever, Rosenbach, H. E. Russell, St. John, Sellew, Shively, Sisam, E. R. Smith, G. W. Smith, I. W. Smith, Stafford, Stouffer, Stowell, J. S. Turner, Wait, R. A. Wells, J. J. Wheeler, F. M. Wright, Wyant, J. M. Young.

This meeting was held in conjunction with the meeting of the Mathematical Association of America and in affiliation with the annual meeting of the A.A.A.S. Of the numerous lectures and papers presented at general sessions and before the various Sections of the A.A.A.S., of interest to mathematicians, no mention can here be made except for the symposium on relativity held before the Physical Society on Wednesday forenoon and the address of Professor D. C. Miller as Retiring President of the American Physical Society on The ether-drift experiment, its history and significance. 
The forenoon and afternoon of Tuesday were used for the reading of the papers listed below. For this purpose the meeting was divided into two sections: During the forenoon the Section on Analysis, presided over by Vice-President T. H. Hildebrandt, heard the papers numbered 1 to and including 14 ; at the same time the papers numbered 15 to and including 23 , and also number 43, were presented before the Section on Algebra, where Professor Carmichael, relieved by Professor Stouffer, occupied the chair. In the afternoon came the Section on Point Sets (papers numbered 24, 25, 26, 27, 28, 42 and 44) which was presided over by Professor R. L. Moore, Chairman of the Southwestern Section, relieved by Professor Hildebrandt, and the Section on Geometry, Mechanics and Applied Mathematics (papers numbered 29 to and including 41) in which Professor Dunham Jackson took the chair. The papers by Bennett, Camp, Coble, Davis, Ford, Ingraham, Kryloff, Nowlan, Shohat, Swingle, and Wilson were read by title. Mr. Messick and Professor Kryloff were introduced to the Society by Professor Dresden; Messrs. Whyburn and Cleveland by Professor R. L. Moore, who also read their paper; Mr. Swingle by Professor R. L. Wilder, and Mr. Goff by Professor Carmichael.

On Wednesday forenoon a joint meeting was held with Section A of the A.A.A.S. and with the Mathematical Association of America under the chairmanship of Professor W. H. Roever, Chairman of Section A. The program for this meeting consisted of two papers: one on The Heine-Borel theorem and allied problems, by Professor T. H. Hildebrandt, who has completed his term of office as Vice-President of the Society; the other on The algebraic numbers and division, by Professor J. C. Fields, the retiring address of the Chairman of Section A. Professor Hildebrandt's paper will appear in full in a later number of the Bulle'tin.

A noteworthy feature of this meeting was the Third Josiah Willard Gibbs lecture on Some modern views of space, delivered at a general session of the A.A.A.S. at 4 P.M. on Wednesday afternoon, by Professor James Pierpont. As this lecture will 
be published in full in an early issue of the Bulletin, no further account of it will be given here. The speaker and the Local Committee on Arrangements must have felt well repaid for their work when they saw an audience of little less than one thousand persons assembled to hear this lecture. The speaker was introduced by Professor E. R. Hedrick.

A very pleasant occasion was the joint dinner of the mathematical organizations at the City Club on Wednesday evening. One hundred twenty-seven persons were present. The toastmaster, Professor F. R. Moulton, and Professors Hedrick, Pierpont, Hughes, Carmichael, and Coolidge, who were called upon to speak, contributed greatly to the success of this function with which the meeting of the Society was brought to a close. A resolution was adopted expressing the appreciation of all those present to Professors Luby and Stouffer and the other local members of the Committee on Arrangements. The Mathematical Association of America continued its sessions throughout Thursday.

Professor Carmichael reported for the Committee, appointed by the Council to arrange for a symposium lecture at the next April meeting of the Society at Chicago (April 2 and 3, 1926), that Professor Dresden would speak on recent work in the calculus of variations.

1. Mr. C. A. Messick: A new method of determining Bernoulli's numbers.

The methods for determining Bernoulli's numbers seem to belong to two classes, one depending on successive differentiation and the other on multiplication of infinite series. The new method is of the second class. The expansion of $x /\left(e^{x}-1\right)$, which has the Bernoulli numbers for coefficients, is multiplied by the expansion of $\left[e^{(l+i) x}-e^{(l-i) x}-e^{i x}+e^{-i x}\right] / 2 i x$; the product, $\sin x$, is also expanded into a power series. Equating of coefficients leads to four equations. From one, the Bernoulli numbers of odd index can be computed, from another, those of even index; the remaining two equations supply a numerical check. The advantage of the method lies in the fact that in the equations here used there are only half as many terms as there are in the equations now in use, so that the arithmetic is much simpler.

An expansion for $\sin x$ is also obtained in terms of Bernoulli's numbers, which is believed to be new. 
2. Professor J. A. Shohat: Asymptotic expression for Jacobi's polynomials derived from a finite-difference equation.

The difference-equation for Jacobi's polynomials corresponding to $(-1,1)$ can be written as follows: $T_{n+2}(x)-x T_{n+1}(x)+\frac{1}{4} T_{n}(x)=$ $\mathrm{O}\left(1 / n^{2}\right)\left[T_{n+1}(x)+T_{n}(x)\right],(n \geqq 1)$, and gives the asymptotic expression of $T_{n}(x)$ (for $n$ very large) by a method similar to that of Liouville (JournaL De Mathématiques, vol. 2, 1837) and capable of extension to more general classes of Tchebycheff's polynomials. The method has some connection with that given by W. B. Ford (Annali di Matematica, (3), vol. 3, and Transactions of this Society vol. 25, No. 3). For, Jacobi's polynomials we use Stekloff's results that $2^{n-1}\left|T_{n}(x)\right|=(\sqrt{n}) \mathrm{O}$ inside of $(-1,1)$ (Memoirs of the Russian Academy of Science, 1917).

3. Professor P. R. Rider: The analysis of a U-shaped frequency distribution.

In graduating a U-shaped frequency distribution, Pearson has been able successfully to employ his curve of Type I. It would seemingly be impossible, or at best extremely unsatisfactory, to fit a curve by the method of Charlier to a distribution of this form. In the present paper, the author has fitted a U-shaped distribution by means of two component Charlier curves of Type B. The fit is better than that obtained by Pearson's method and the computation involved is very considerably less.

\section{Professor E. L. Dodd: On certain averages in periodogram} analysis.

Let $A v\left(u_{t}\right)$ denote the limit of the average of consecutive terms in an infinite sequence. An addition theorem is proved for trigonometric series. This is applied to periodogram analysis where $s$ variates are arranged in $k$ columns to test for a period of $k / l$, with $k / l=2 \pi / \theta$. It leads to the consideration of a triangular array of products, with $u_{t} u_{t+r}$ in the $(t+1)$ th row and the $(r+1)$ th column, where $t+r \leqq n$, the number of variates available. The double averages of these columns may be called graduated values of $u_{t}$ in the sense that periods are left invariant, while amplitudes are squared and phase differences at the origin are obliterated.

5. Professor E. V. Huntington: Sets of independent postulates for four types of means.

The four types of means or averages considered in this paper are the arithmetic mean, the geometric mean, the harmonic mean, and the rootmean-square. The postulates presented are the conditions which a function of $n$ variables, $f\left(x_{1}, x_{2}, \cdots, x_{n}\right)$, must satisfy in order to coincide with one or another of these means-several sets of independent postulates being given for each of the four types. Reference is made to Schimmack's postulates for the arithmetic mean (Mathematische Annalen, 1909), and to R. D. Beetle's discussion of the complete independence of Schimmack's postulates (Mathematische AnNalen, 1915). 
6. Professor O. Dunkel: The alternation of the nodes of linearly independent solutions of second order differences equations.

A solution $u(n)$ of the difference equation $u(n+2)=A(n) u(n+1)$ $-B(n) u(n), B(n)>0$, is plotted against the integral values of $n$ and the points so obtained are connected by segments of a straight line. The points where this broken line cuts the $x$-axis are called the nodes of this solution. Proofs of the theorem that the nodes of two linearly independent solutions separate one another have been given by Porter, Annals of MatheMATICS, (2), vol. 3, 1901-1902, p. 65, and by E. J. Moulton, Ibid., vol. 13, 1911-1912, p. 137. A more direct and simpler proof of this theorem is here given by writing the two functions of $x$ which give the heights of the two broken lines. The wronskian of these two functions is then easily shown to retain the same sign, and from this fact the theorem then follows by the reasoning usual for the similar theorem in differential equations.

7. Professor W. C. Brenke: On the summability of a class of series of Legendrian polynomials.

In this paper the author proves the following theorem: The series of Legendrian polynomials $\Sigma_{0}^{\infty} a_{n} X_{n}$ is summable $(C 1)$ if the coefficients $a_{n}$ form an arithmetic progression. The value of the series is $\left(3 a_{0}-a_{1}\right) h(x)$ where $h(x)$ is the sum of a uniformly convergent series of Legendrian polynomials and is independent of the coefficients $a_{n}$.

8. Professor L. S. Dederick: Successive derivatives of a function of several functions.

Bruno's formula for the successive derivatives of a function of one function is here generalized to a function of several functions. An algebraic property of the new formula is then developed by which any product of higher derivatives of the inner functions occurring in the total derivative of one order is shown to have a coefficient (involving only first order derivatives) which is simply related to those terms of a previous total derivative involving only first order derivatives of the inner functions. Certain theorems are then derived which are useful in the study of higher singularities of transformations of two variables.

9. Professor L. S. Dederick: Classification of singularities of transformations of two variables.

This paper deals exclusively with what are strictly point singularities, i.e., those characterized by the values of a finite number of partial derivatives of the defining functions at one point. The classification is based mainly on the transformation of the ordinary derivative and proceeds according to three indices. The first is the order of the lowest non-vanishing partial derivative of the defining functions. The second is the number of linear factors common to numerator and denominator of the transformed first derivative. These depend only on the transformation and the point 
considered. At any singular point there may be any number of critical slopes, not exceeding the value of the second index. For each of these there is a third index depending on the number of orders of derivatives having critical values. These indices are all invariant under non-singular transformations of either pair of variables. Singularities having the same values of all three indices, however, are not in general equivalent under any nonsingular transformation.

10. Professor Dunham Jackson: On tensors of the second rank in function space. Preliminary communication.

If $f(s)$ is an arbitrary function in an interval $(a, b), \varphi(y)$ a given function of its argument, and $K(x, s)$ a given function for $a \leqq x \leqq b, a \leqq s \leqq b$, suitable hypotheses of continuity being imposed in each case, the expression $\Phi(x)=\int_{b}^{a} K(x, s) \varphi[(f(s)] d s$ may be regarded as defining a vector pointfunction in function space. If $\left[u_{i}(x)\right],\left[v_{k}(x)\right]$ are two complete orthogonal systems on $(a, b)$, and if

$$
f(x)=\sum_{i} a_{i} u_{i}(x)=\sum_{k} b_{k} v_{k}(x), \quad \Phi(x)=\sum_{i} A_{i} u_{i}(x)=\sum_{k} B_{k} v_{k}(x),
$$

purely formal manipulation indicates that the derivatives $\partial A_{i} / \partial a_{j}, \partial B_{k} / \partial b_{l}$ are related by the equations of transformation appropriate to a tensor of the second rank. The invariant resulting from the contraction of the tensor is the "divergence" to which reference has been made in recent communications to the Society, while the tensor itself is related to the notion of curl in ordinary vector analysis. The present paper carries through the proofs of convergence that are needed to supplement the formal calculation, under hypotheses which are very highly specialized, but still retain a significant degree of generality. The tensor property is found also in a variety of other cases, including some for which the divergence is not defined.

11. Professor N. Kryloff: On a method for the approximate integration of differential equations.

The author discusses the approximate solution of the differential system (1): $L(y)=y^{\prime \prime}+A(x) y=f(x) ; y(a)=y(b)=0$, by the method of least squares based upon the minimizing of the integral $\int_{a}^{b}\{L(y)-f(x)\} d x$, and proves that the approximating functions $y_{m}$ tend towards the solution $y$ of the system (1) in virtue of the inequality $\left|y-y_{m}\right| \leqq(b-a)^{3 / 2} \sqrt{\epsilon_{m}}$, where $\epsilon_{m} \rightarrow 0$. He also shows that $y^{\prime}{ }_{m}$ tends toward $y^{\prime}$ and determines the order of magnitude of $\left|y^{\prime}-y_{m}^{\prime}\right|$ and of $\left|y-y_{m}\right|$ according to the restrictive conditions imposed upon $A(x)$ and $f(x)$. The method can be applied to systems more general than (1) and is of importance for practical applications.

12. Dr. C. C. Camp: A method for accelerating the convergence in the process of iteration.

If the convergence of the sequence of iterated values from $f_{1}\left(x_{k}\right)=$ $f_{2}\left(x_{k-1}\right)$ is "exasperatingly slow," a third value $\bar{x}_{k+1}$ may be found by the formula $\bar{x}_{k+1}=x_{k-1}+\left(x_{k}-x_{k-1}\right) /(1-m)$, in which $m=f_{2}^{\prime}\left(x_{k}\right) / f_{1}^{\prime}\left(x_{k}\right)$. This 
amounts to an interpolation or extrapolation suggested by the approximate relation between the increments of $x_{k}$ and $x_{k-1}$. The substitution of $x_{k}$ for $x_{k-1}$ in the numerator of $m$ not only simplifies it but probably leads to a more powerful convergence still. Geometrically the value $\bar{x}_{k+1}$ is found from the intersection of a tangent and a secant, respectively, for the curves $y=f_{1}(x)$ and $y=f_{2}(x)$. The tangent is drawn at $x=x_{k}$ on the former and the secant at $x=x_{k-1}$ on the latter with the slope $f_{2}^{\prime}\left(x_{k}\right)$. Although it differs in two important particulars from Newton's method, the new formula is more direct and, curiously, has as great and probably a greater power of diminishing the number of approximations needed. The author is extending the method to systems in two or more unknowns.

\section{Professor H. T. Davis: Asymptotic solutions of linear} differential equations of infinite order with constant coefficients.

This paper generalizes work of F. Schürer on non-homogeneous linear differential equations of infinite order with constant coefficients by considering the case where the given function is expressible in a Laurent series. The method of inverse operators is used to obtain a formal solution which is, in general, divergent. This expansion is then shown to be the asymptotic representation of the solution of the equation and Borel's method of summation is employed to obtain its integral form. Such equations as $u(x+1)-u(x)=1 / x$ are easily solved by the method of this paper.

14. Professor T. A. Pierce: An approximation to the least root of a cubic equation, with application to finding units in cubic fields.

This paper gives a method for approximating the least root of a cubic equation. The method is somewhat similar to Bernoulli's method of approximating the greatest root of an equation. Application is made to the problem of finding units in certain pure cubic number fields.

\section{Professor F. S. Nowlan: Representation of integers by} certain ternary cubic forms.

This paper will appear in full in an early issue of this BulletiN.

\section{Professor N. R. Wilson: Integers and basis of a number} field.

The existence theorems of the standard theory of algebraic numbers do not always lead to very practicable methods of computation in individual cases. Such methods of computation form the main subject of this paper. It is first shown that the field can be expressed in terms of a special finite set, named maximal reduced integers, the set combining easily into a basis. The usual theorems about a basis and a theorem connecting the discriminants of the field and defining equation follow as corollaries. Certain 
other relations to facilitate computation are then obtained, the whole illustrated by application to the general cubic.

\section{Professor O. C. Hazlett: On formal modular invariants for the general binary form with respect to $G F\left(p^{n}\right)$.}

This paper considers the general binary form of order $m$ and its formal modular invariants under the total linear group with coefficients in the general Galois field. It is proved that a formal, modular invariant of $f(a ; x)$ is congruent to a polynomial (or root of such a polynomial) in the algebraic invariants of any $m+1$ forms of the system consisting of $f(a ; x)$ and certain related forms $f\left(a^{p^{n}} ; x\right), f\left(a^{p^{2 n}} ; x\right)$, and the modular invariants of the total linear group on the $m+1 a$ 's. This suggests dividing formal, modular invariants of $f$ into $m+1$ classes. Then simple annihilators for formal, modular invariants of each class are found, which are consistent with the complicated annihilators for the general formal, modular invariants of $f$ obtained by Dickson for special cases. Since any algebraic invariant of a system of forms, $f, g, \cdots$, is an intermediate invariant of $f+k g$ $+\cdots$, we have a general method of obtaining from the algebraic invariants of $f$ the formal modular invariants and covariants. In particular, we obtain the formal modular invariants of three or more pairs of variables from the formal modular invariants of two pairs for the general Galois field.

\section{Professor E. B. Stouffer: A simple derivation of Kro- necker's relation among the minors of a symmetric determinant.}

Kronecker's linear relation among the minors of a symmetric determinant has been proved in several ways. In the present note Professor Stouffer derives the relation in a very simple and elementary manner by the use of certain differential operators.

19. Professor J. S. Turner: Rational triangles in which one angle is a rational multiple of another.

In a former paper, a method was given for finding the relation between the sides of a triangle $A B C$ when $A=n B$, where $n$ is rational; and the complete integral (and also rational) solution was given when $n=2,3,4,5,3 / 2$. In the present paper, a geometric method of solution is described, and the complete solution is given when $n=l / m$, where $l+m=7$ or 8 .

20. Professor C. H. Sisam: On the quinquenary cubic expressible as the sum of seven cubes.

It is well known that a cubic in five variables can be expressed as the sum of seven cubes only if a certain invariant vanishes although a crude counting of constants seems to indicate that this restriction is unnecessary. The purpose of this paper is to determine this invariant explicitly and to study some properties of the cubic varieties for which this invariant vanishes. 
21. Professor W. E. Edington: Groups generated by operators whose transforms are equal in pairs.

If a set of $m$ independent generators is subjected to the condition that for every pair $s_{i}^{-1} s_{i} s_{j}=s_{j}^{-1} s_{i} s_{j}, i \neq j, i, j=1,2, \cdots, m$, the orders of the generators will be equal, say $n$, and $s_{j}^{-1} s_{i} s_{j}^{-1} s_{i}=\left(s_{i} s_{j}^{-1}\right)^{k+1}$ where $(k+1)^{n} \equiv 1$, $\bmod (2 k+1)$. This last condition is satisfied only when $2 k+1$ is a divisor of $2^{n}-1$. For two generators the groups are of order $n(2 k+1)$ and one or more groups exist for every value of $n>1$. For three generators when $n$ is even and $2 k+1=2 l+1=3$, the groups are of order $3 n \alpha^{2}, \alpha=2,3, \cdots$. For $m$ generators, $m>2$ if $2 k+1=2 l+1=\cdots=2^{n-1}-1$, the order of the groups generated is $n(2 k+1)$, provided a special commutativity assumption is made. The theory of the generators as substitutions is developed so that the generators for any of the desired groups may be written down, thus proving the existence of the groups.

22. Professor M. H. Ingraham: Note on the extension of groups to obtain nth roots.

In this paper the following theorem is proved. For every group $G$ there exists the group $G_{1}$ containing a subgroup isomorphic with $G$, and such that for every integer $n$ and element $g_{11}$ in $G_{1}$ there exists an element $g_{12}$ in $G_{1}$ such that $g_{12}^{n}=g_{11}$.

23. Dr. R. G. Lubben: Concerning the separation of point sets by curves.

In this paper the author proves the following theorem. If $K$ and $H$ are bounded continua having in common a totally disconnected point set $T$, then a necessary and sufficient condition that there exist a simple closed curve containing $T$ which separates $H-T$ is as follows: If $P$ is a point of $T$ and $C$ is a circle with center at $P$, there exists a circle $C_{1}$ with the same center, such that if $x$ and $y$ are any two points of $H-T$ from $K-T$ within $C_{1}$ there exists a simple continuous arc within $C$ which contains $x$ and $y$, but no point of $K$; and similarly, if $z$ and $w$ are two points of $K-T$ within $C_{1}$, there exists an arc within $C$ containing them but no point of $H$.

24. Mr. G. T. Whyburn and Mr. C. M. Cleveland: $A$ characterization of certain domains by properties of their boundaries.

The following theorems are established:

I. A domain $D$ has property $S($ R. L. Moore, Fundamenta MatheMATICAE, vol. 3, p. 232) if, and only if, (a) every maximal connected subset of the boundary of $D$ is a continuous curve, (b) there are not more than a finite number of these continuous curves of diameter greater than any preassigned positive number.

II. A domain $D$ is uniformly connected "im kleinen" with reference to every one of its bounded subsets, if and only if, (a) every maximal connected 
subset of its boundary is either a point, a simple closed curve, or an open curve, (b) $J$ being any simple closed curve and $e$ a positive number, there are not more than a finite number of maximal connected subsets of the boundary of $D$ which have points within $J$ and are of diameter greater than $e$.

III. A bounded domain $D$ of connected boundary $B$ becomes uniformly connected im kleinen upon the addition of a point $O$ if, and only if, (a) every maximal connected subset of $(B-O)$ plus $O$ is a simple closed curve, (b) there are not more than a finite number of these curves of diameter greater than any preassigned positive number.

\section{Professor R. L. Moore: Indecomposable continua.}

It is shown that various internal properties of all bounded continua that contain no subsets separating the plane are also properties of every bounded indecomposable continuum which does separate the plane into just two domains and which is the boundary of each of these domains. In particular, it is proved that if $M$ is either (a) a bounded continuum no subset of which separates the plane, or (b) any indecomposable bounded continuum (whether it separates the plane or not) and $G$ is a countable number of subcontinua of $M$ no one of which separates $M$, then $M$ is not separated by the sum of all the continua of the set $G$ (in case $M$ is an indecomposable continuum the hypothesis that no element of $G$ separates $M$ is always satisfied). The author raises the question whether there exists an indecomposable continuum which separates the plane but which is in one to one continuous correspondence with some one which does not separate the plane.

\section{Professor E. W. Chittenden: On the classification of} points of accumulation in the theory of abstract sets. Preliminary communication.

In the theory of abstract sets the points $p$ of accumulation of a set $E$ fall into two classes: those with the property that every neighborhood of $p$ contains a point of $E$ distinct from $p$ as an interior point, and those for which this condition is not satisfied. The points of the first kind will be called proper points of accumulation, those of the second, improper. The paper considers the fundamental properties of abstract sets in relation to this classification.

\section{Mr. P. M. Swingle: An unnecessary condition in two} theorems of analysis situs.

R. L. Moore (Transactions of this Society, vol. 17 (1916) pp. 131164, Theorem 48) has shown that the following theorem of Schoenfliess (GötTINGER NACHRICHTEN, 1902, p. 185) is true in a space $S$ satisfying his set of axioms $\Sigma_{3}$ : if $K$ is a closed, bounded point set such that $S-K=S_{1}+S_{2}$ and (1) if every two points of $S_{i}(i=1,2)$ can be joined by a simple continuous arc contained in $S_{i}$; (2) if every arc joining a point of $S_{1}$ to a point of $S_{2}$ contains at least one point of $K$ : and (3) if every point $P$ of $S_{i}(i=1,2)$ 
and every point $Q$ of $K$ can be joined by an arc $P Q$ such that $P Q-Q$ is contained in $S_{i}$, then $K$ is a simple closed curve. J. R. Kline (TransacTIONS of THIS Society, vol. 18 (1917), pp. 177-184) proved that if $K$ is a closed point set such that $S-K=S_{1}+S_{2}$ where $S_{i}$ are unbounded and if conditions (1), (2), and (3) hold, then $K$ is an open curve. In this paper it is shown that both these conclusions hold if condition (1) is omitted.

\section{Professor A. D. Campbell: Pencils of conics in the} Galois fields of order $2^{n}$.

The author derives the classes of pencils of conics in the Galois fields of order $2^{n}$, and gives a typical pencil of conics for each class. He divides the pencils into four sets, as follows: those with double lines, those with no double lines but with real line pairs, those with no double lines and no real line-pairs but with conjugate imaginary line pairs, those with no degenerate conics at all. In these fields every number is a square and has just one square root; $(a x+b y+c z)^{2}=a^{2} x^{2}+b^{2} y^{2}+c^{2} z^{2}$; and the discriminant of the general conic $a x^{2}+b y^{2}+c z^{2}+f y z+g z x+h x y=0$ is $f g h+a f^{2}+b g^{2}+c h^{2}$. There exist pencils like $\lambda x^{2}+\mu y^{2}=0$ that consist entirely of double lines.

\section{Professor A. D. Campbell: Plane cubic curves in the} Galois fields of order $p^{n}, p>2$.

The author derives the different classes of cubic curves in the Galois fields of order $p^{n}, p>2$ and gives a typical cubic curve for each class. There exist both singular and non-singular cubic curves without real points of inflection, also cubics whose points of inflection have harmonic polars that cut the cubic only in imaginary points. The equations of condition for real points of inflection are derived; they differ according as $p=3$ or $p>3$. If $p=3$ a cubic curve can have at most only three points of inflection. Furthermore, peculiarities occur according as -1 is or is not a perfect square in the field, and according as the field does or does not contain non-cubes

30. Professor R. D. Carmichael: Representation of the finite projective geometries $P G\left(k, p^{n}\right)$ by means of abelian groups.

This paper exhibits a representation of the finite projective geometries $P G\left(k, p^{n}\right)$ by means of abelian groups of order $p^{(k+1) n}$ and type $(1,1,1, \cdots)$, where $p$ is a prime number. In this representation a selected set of subgroups of order $p^{n}$ constitute the "points" of the finite geometry. By means of this representation the main part of the theory of the finite geometries is translated into a corresponding part of the theory of Abelian groups.

31. Professor R. D. Carmichael: Groups of isomorphisms of abelian groups of type $(1,1,1, \cdots)$.

By means of the marks of a Galois field a variety of analytical representations are given of the group of isomorphisms of Abelian groups of type $(1,1,1, \cdots)$ and of certain of its subgroups, in which certain previously known representations are included as special cases. 
32. Professor R. D. Carmichael: Collineation groups in the finite geometries.

Various subgroups of the general collineation group in the finite projective geonetries $P G\left(k, p^{n}\right)$ are treated with special reference to the problem of constructing primitive groups. In particular, various classes of doubly transitive and of triply transitive groups are exhibited, which are contained as subgroups in the general collineation group.

\section{Professor Louis Ingold: Generalizations of curl and divergence.}

In this paper the curl and the divergence of a vector in three-dimensional space are expressed in invariant form by means of Maschke's symbolic method. These forms are then extended to $n$ dimensions. The list of extensions includes (1) a series of forms $C_{k}$ analogous to the curl of a vector, and (2) a series of forms $L_{k}$ analogous to the Lorentzian of a tensor of the second order in four dimensions. The divergence of a vector belongs to this last series and may be denoted by $L_{0}$. In the case of special tensors which may be regarded as simple $k$-dimensional vectors of the space, it is found that the two series of operations are not independent.

\section{Professor Louis Ingold: Tensors and symbolic invariants.}

In Maschke's symbolic method for representing invariants of a quadratic differential form $A=\Sigma g_{i j} d x^{i} d x^{i}$, the coefficient $g_{i j}$ is represented as a product of symbols $f_{i} f_{j}$ or equivalently as $\varphi_{i} \varphi_{j}$ or $\psi_{i} \psi_{j}$ etc. In this theory there are invariants in which certain symbols occur just once as factors in each term. In this paper it is shown that symbolic invariants of the form $\Sigma Q^{\alpha_{1}} \cdots \alpha_{k} \varphi_{\alpha_{1}}^{1} \cdots \varphi_{\alpha_{k}}^{k}$ where the $\varphi^{1}, \cdots, \varphi^{k}$ are distinct symbols, are tensors whose contravariant components are the $Q^{\alpha_{1}} \cdots \alpha_{k}$. The converse is also true. Other symbolic invariants contain derivatives of the $\varphi^{j}$. These are not included in the usual tensor theory. The paper also contains an extension of Maschke's fundamental theorem, which makes it possible to apply Maschke's methods to a very general class of vectors and tensors.

35. Dr. Theodore Bennett: Mapping by means of linear systems of curves invariant under Cremona involutions.

This paper studies the mapping of pairs of copoints in the Geiser, Bertini, and Jonquière involutions upon points of a plane or quadric. The map, $J^{\prime}$, of the locus $J$ of fixed points is the plane quartic, and the sextic of genus four on a quadric cone, in the Geiser and Bertini involutions respectively. In the Jonquière involution of orders $2 k-1$ or $2 k$ respectively, $J^{\prime}$ is a curve with characteristic $(2 k-2,2)$ on a quadric, or a plane curve of order $2 k$ with a $(2 k-2)$-fold point. Systems of contact curves and surfaces of $J^{\prime}$ are enumerated corresponding to systems of composite invariant curves. In the Geiser involution the lines which carry pairs of coincident copoints form an envelope $E_{4}$, those joining pairs of copoints on a composite invariant curve form a contact envelope of $E_{4}$. Thus the systems 
of contact envelopes of $E$ are obtained as the envelopes perspective to systems composite invariant curves. If quintics of a pencil have six common double points and one simple point at seven points forming an Aronhold set of double points of a quartic envelope $E_{4}$ then the conics perspective to them form a quadratic system, and are contact conics of $E_{4}$.

36. Professor N. Altshiller-Court: On two intersecting spheres.

Generalizing a property of the plane (this Bulletin, vol. 29 (1923), p. 442) it may be shown that the cone determined by the common circle of two intersecting spheres and a variable point on one of these spheres meets the second sphere again along a circle whose diameter is constant. The length $x$ of this diameter is given by the proportion $x: d=s: p$, where $d$ is the diameter of the common circle of the two spheres, $s$ their line of centers, and $p$ the radius of the first sphere.

37. Professor A. B. Coble: Double binary forms with the closure property.

Two examples of double $(3,3)$ binary forms with the closure property have been given by Professor Louise Cummings. The grouping of the allied configurations is one that can be extended in two ways. It is the purpose of this paper to prove that these more general configurations are associated with poristic forms which in all cases are factorable. After the elimination of these somewhat degenerate cases, the $(3,3)$ form $(a \tau)^{3}(\alpha t)^{3}$ is again considered and all the forms which close with a set of $n t$ 's and $n \tau$ 's are determined for values of $n<10$.

38. Professor L. R. Ford: On motions which satisfy Kepler's first and second laws.

This paper contains an investigation of central forces depending upon the distance and the velocity for which all orbits are conics with one focus at the center of attraction. The most general such force, $\varphi$, is given by the formula $F\left(\varphi r^{2}, v^{2}-2 r \varphi\right)=0$, where $r$ is the distance of the body from the center of attraction, $v$ is its velocity, and $F$ is an arbitrary function. Kepler's third law can be replaced by any functional relation between the period and the semi-major axis and the force is thereby determined.

39. Professor R. D. Carmichael: Transformations leaving invariant certain partial differential equations of physics.

The generators are determined of the general group of point transformations which leave invariant the partial differential equation of wave motion in $k$ dimensions. A similar problem is treated in the case of certain other (and related) partial differential equations of physics.

40. Mr. J. A. Goff: Transformations leaving the heat equation invariant.

Employing the methods and results of the preceding paper by Professor Carmichael, the author determines the generators of the general group of 
point transformations which leave invariant the heat equation in the case of a space of $n$ dimensions.

41. Dr. R. G. Lubben: The separation of mutually separated subsets of a continuum by curves.

If $T$ is a closed, totally disconnected subset of a bounded continuum $K$; if $K=K_{1}+K_{2}, K_{1}$ and $K_{2}$ are mutually separated and have points $x_{1}$ and $x_{2}$ respectively, then there exists a simple closed curve $J$ which contains in common with $K$ only points of $T$ that are limit points of $K_{1}$, and which separates $x_{1}$ and $x_{2}$. If the condition that $K$ be bounded be removed from the hypothesis, a curve $J$ exists, either a simple closed curve or an open curve. We have the following corollaries: If $P$ is a cut point of a bounded continuum $M$, there exists a simple closed curve which contains only $P$ in common with $M$, and which separates $M-P$; hence $P$ is accessible from $S-M, S$ being the set of all points. If the closed point set $M$ is the sum of points belonging to an unbounded collection of continua, $M=$ $M_{1}+M_{2}$, where $M_{1}$ and $M_{2}$ are mutually separated point sets, $x$ and $y$ are points in $M_{1}$ and $M_{2}$ respectively, then there exists an open curve which separates $x$ from $y$ and contains no point of $M$.

42. Professor O. C. Hazlett: Note on the arithmetic of an associative algebra over any algebraic field.

This paper is an addendum to the author's paper read before the Toronto Congress, and (without using normalized units except for a nilpotent algebra or the notion of character) proves that if $A$ is any associative algebra over any algebraic field, then the characteristic determinant for the general number $x$ of $A$ has the same irreducible factors as the characteristic determinant of the component of $x$ in $S$, whose $S$ is any semisimple algebra contained in $A$. From this it follows that the arithmetic of $A$ is associated with the arithmetic of $S$.

43. Professor J. R. Kline: A characterization of continuous curves every subcontinuum of which is a continuous curve.

The author shows that a necessary and sufficient condition that every sub-continuum of a two-dimensional continuous curve $M$ be a continuous curve is that every irreducible continuum joining two distinct points $A$ and $B$ of $M$ be a simple continuous arc from $A$ to $B$.

E. B. Stouffer, Secretary of the Southwestern Section. Arnold Dresden, Assistant Secretary of the Society. 